Heylen, E., Deconinck, G., Van Hertem, D. (2016). Analysis Framework for Performance Evaluation of Reliability Management in Power Systems with Increased Uncertainty.

This is the author's version of an article that has been accepted for publication in Conference proceedings of The annual European Safety and Reliability Conference 2016 (ESREL 2016). Changes are made to this version by the publisher prior to publication.

(C)2017 Taylor \& Francis Group, London: Personal use of this material is permitted. Permission from Taylor \& Francis Group, London must be obtained for all other uses, including reprinting/republishing this material for advertising or promotional purposes, creating new collective works for resale or redistribution to servers or lists, or reuse of any copyrighted components of this work in other works. 


\title{
Analysis Framework for Performance Evaluation of Reliability Management in Power Systems with Increased Uncertainty
}

\author{
E. Heylen, G. Deconinck \& D. Van Hertem \\ Div. ELECTA, Dept. Electrical Engineering, KU Leuven \& EnergyVille, Leuven, Belgium
}

\begin{abstract}
Power system reliability is managed according to a reliability criterion, which determines the acceptable reliability level in the system. However, due to increasing uncertainties in power systems, the currently used deterministic $\mathrm{N}-1$ reliability criterion is challenged and alternative probabilistic reliability criteria are sought for. These alternatives take uncertainty into account in a more convenient way, thereby outperforming deterministic approaches. Evaluating and quantifying performance of new power system reliability criteria and comparing these to the state-of-the-art approach are crucial to give incentives to stakeholders to change the manner in which they do their reliability management. Any alternative must be proven to be cost-effective over the entire operating range. In this paper, an analysis framework is specified that allows the development of techniques for performance evaluation of reliability management according to various power system reliability criteria. A hybrid technique for evaluating performance of short term reliability management, combining aspects of simulation techniques and analytical contingency enumeration, is presented and compared with an analytical state enumeration approach in a quantitative and qualitative way. Both techniques are applied to a 5 node test system for three uncertainty levels leading to similar results in terms of expected total system cost.
\end{abstract}

\section{INTRODUCTION}

Economics, social well-being of a modern society and the quality of life are strongly influenced by reliability of electricity supply. An adequate reliability level, which balances the value of reliability and its cost, is required in one of the most critical infrastructures of modern society. Moreover, the power system is complex and continuously evolving, while the currently used reliability management based on the deterministic N-1 reliability criterion was developed with a centrally planned and operated nature of generation, transmission and distribution in mind ( $\mathrm{Al}-$ lan \& Billinton 2000). The deterministic N-1 criterion is challenged by the increasing penetration of renewable energy sources in the system, which has led to higher uncertainties and less cost-effective system operation, as larger reliability margins are required. Furthermore, a N-1 criterion only considers a limited set of contingencies, i.e. unexpected outage or failure of a system component such as a line, generator or transformer, and deems other contingencies as not probable. Nevertheless, reports show that major disturbances occur due to combinations of failures not dealt with in the currently used deterministic approach based on a N-1 criterion (Phadke \&

${ }^{*}$ The work of E. Heylen is funded by Research Foundation Flanders (FWO)

The research leading to these results has received funding from the European Union Seventh Framework Programme under Grant Agreement No 608540.
Thorp 1996, Tamronglak, Horowitz, Phadke, \& Thorp 1995). Consequently, the implementation of alternative probabilistic reliability criteria needs to be considered (CIGRE Working Group C4.601 2010). Alternative probabilistic approaches able to overcome challenges and shortcomings of deterministic reliability criteria are under development ${ }^{1}$. In order to give incentives to each stakeholder in the power system to change their manner of reliability management, it is important to quantify benefits of using alternative reliability criteria.

Techniques for evaluating and quantifying performance of power system reliability management according to various reliability criteria are not extensively covered in literature. Within the framework of the GARPUR project ${ }^{1}$, a quantification platform is developed that allows to simulate TSOs' short term reliability management according to various reliability criteria, evaluate the final system state and reliability decision related trajectory and compare the performance of various reliability criteria (Heylen, Labeeuw, Deconinck, \& Van Hertem 2016). In order to make an honest comparison, an appropriate performance evaluation technique is important. Similarities can be found between probabilistic reliability or security assessment methods (Warwick 1997, Allan \& Billinton 2000, Meliopoulos 2005) and performance evaluation techniques, but there are some important

\footnotetext{
${ }^{1}$ www.garpur-project.eu
} 
differences between them.

In this paper, an analysis framework is specified that allows the development of techniques for performance evaluation of reliability management according to various power system reliability criteria. A hybrid technique for evaluating performance of short term reliability management, following this analysis framework, is presented. This technique combines aspects of simulation techniques and analytical contingency enumeration. The hybrid technique is compared with an alternative analytical state enumeration approach in a qualitative and quantitative way. This comparison highlights important aspects to consider in order to evolve towards an optimal balance between accuracy and computational complexity of performance evaluation techniques.

Section 2 defines the analysis framework. The hybrid technique following the analysis framework is presented in section 3 , as well as the analytical approach used for comparison. Both techniques are applied to a 5 node test system that is managed according to two different reliability criteria. The case study and the comparison of techniques are included in section 4 . Section 5 concludes the paper.

\section{PERFORMANCE EVALUATION OF POWER SYSTEM RELIABILITY MANAGEMENT}

In order to assess performance of power system reliability management according to a particular reliability criterion, different aspects need to be considered. The analysis framework consists of:

- Definition of performance of reliability management and which aspects to include

- Definition of system boundaries to limit the extent of the analysis

- Selection of the level of modelling detail and analysis method

- Selection of performance indicators

Those aspects are handled more in detail in following subsections.

\subsection{Performance of reliability management}

The ideal reliability criterion according to which reliability can be managed is the one maximizing socioeconomic surplus ${ }^{2}$. It results in an optimal balance between reliability cost and socio-economic criticality as indicated by the dashed line in figure 1. However, using such a criterion in practice for decision making is not possible due to data limitations. Alternatively to deterministic reliability criteria used so far, limits

\footnotetext{
${ }^{2}$ Socio-economic surplus is the sum of surplus or utility of all stakeholders, including external costs and benefits (e.g. environmental costs) over the expected operating range (GARPUR consortium 2016)
}

on quantitative reliability indices might be used that approximate reliability management according to the maximal socio-economic surplus criterion.

An ex-post evaluation is required to assess performance of power system reliability criteria. Performance of power system reliability management according to various reliability criteria needs to be comparable for various types of reliability criteria, e.g. deterministic or probabilistic in nature, defined in terms of different (types of) acceptability constraints, etc. A unified performance evaluation of power system reliability management consists of evaluating utility cost versus socio-economic criticality. Criticality refers to the extent of consequences for users of an infrastructure when a system does not carry out its intended function (Kjølle, Gjerde, \& Hofmann 2013). Criticality describes how severe the consequences are for users who are dependent on the system. Consumer interruption costs (Allan \& Billinton 2000) and environmental costs are examples of socio-economic criticality. Utility cost is the cost for achieving a particular reliability level. The principle of utility cost versus socio-economic criticality is graphically illustrated in figure 1. Benchmarking results of alternative reliability management strategies with the currently used N-1 approach is a good practice to evaluate how alternative reliability management strategies perform compared to the state-of-the-art approach.

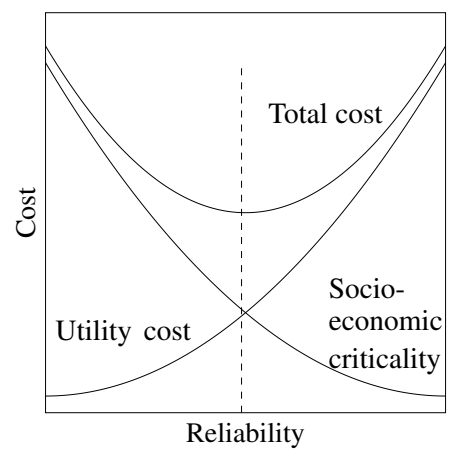

Figure 1: Utility cost versus socio-economic criticality

\subsection{System boundaries}

The system in performance evaluation of reliability management is defined by three main attributes ${ }^{3}$ (GARPUR consortium 2016): (1) the stakeholders included in the evaluation, i.e. TSO, consumers, generators, etc., (2) the area and (3) the time over which the performance evaluation is made. The time horizon of the reliability management strategy under evaluation is also an important aspect to define. The three main time horizons corresponding to different TSO decision making processes are long term, i.e. system development, mid term, i.e. asset management and short

\footnotetext{
${ }^{3}$ A partial equilibrium approach is used which implies that changes in the electricity market do not have a significant effect on other markets (GARPUR consortium 2016)
} 
term, i.e. operational planning and real time operation. The decision making processes are interlinked.

\subsection{Analysis methods and level of modelling detail} Performance evaluation of power system reliability criteria consists of three main tasks:

1. Generation of sample set of states characterized by forecasts of power generation (incl. Renewable Energy Sources (RES)) and load, real time realization of RES power generation and load and contingencies.

2. Simulation of reliability management according to a particular reliability criterion for the states in the sample set.

3. Post-processing of simulation results according to the type of sample set.

Similarities can be found between reliability assessment techniques and performance evaluation techniques for power system reliability management. Performance of reliability management is related to the resulting reliability level in the system. Reliability assessment techniques evaluate whether the system is reliable in a particular system state and therefore verify whether the specified reliability criterion is satisfied or not. Reliability assessment of the currently used deterministic $\mathrm{N}-1$ reliability criterion only focuses on up to $\mathrm{N}-1$ contingency states and omit higher order contingency cases. Stochastic behaviour of load and RES is also not considered in those approaches. Performance evaluation techniques on the other hand should in theory assess all possible system states in terms of contingencies and load and RES realizations in order to make an overall evaluation of performance, similar to probabilistic reliability assessment. This is not doable in practice, especially not in large power systems. Appropriate systems states to evaluate need to be selected without favouring a particular reliability criterion. Contingency cases can be selected based on probability of occurrence or the risk they are subject to (Singh \& Mitra 1997, Khan \& Billinton 1992, Stefopoulos, Yang, Cokkinides, \& Meliopoulos 2005). Uncertainty in load and RES can be represented by a multi-dimensional uncertainty cloud, which includes (cross-) correlations. In order to make an honest comparison of performance of various reliability criteria, it is important that values of exogenous variables are the same for all reliability criteria under evaluation and that the same performance indicators are used, which hold for both deterministic and probabilistic reliability criteria. An important difference with reliability assessment methods is that reliability assessment mainly focuses on the evaluation of the final system states, while performance evaluation requires that also the reliability decision related trajectory is evaluated. This is crucial as different reliability criteria imply different decisions for equal system conditions
(Heylen, Labeeuw, Deconinck, \& Van Hertem 2016), as they consider uncertainty in different ways.

Two fundamentally different methods for performance evaluation of power system reliability management exist similarly to reliability assessment: simulation and analytical techniques. Sample generation and processing of simulation results differ for different methods. Simulation techniques, often referred to as Monte Carlo simulation, simulate the actual process and random behaviour of the system. States are randomly selected based on their probability resulting in high probability states that are simulated more likely and possibly several times. Analytical methods, such as analytical state enumeration, determine performance indicators based on a mathematical model. States are selected based on probability and/or contingency order and are evaluated only once. While simulation techniques allow to determine probability distributions of performance indicators, analytical approaches mainly focus on expected values (Allan \& Billinton 2000). Hybrid techniques combine aspects of both methods. The required type of information is important to consider in the development or selection of a performance evaluation technique.

The optimal level of modelling detail, balancing accuracy and computational complexity, depends on the considered time horizon of reliability management and is subject to further study. The level of modelling detail determines the required type of samples, e.g. sequential versus non-sequential samples, and the simulation approach, e.g. should an AC or DC power flow be used for the simulation of TSO's reliability management.

\subsection{Performance indicators}

The ideal socio-economic performance indicator of power system reliability management is total socioeconomic surplus, because it covers overall costs and benefits of different system stakeholders. Data to calculate socio-economic surplus are not always available. The sum of reliability and interruption costs is a good approximation for socio-economic surplus under two simplifying assumptions ${ }^{4}$ (GARPUR consortium 2016):

- Changes in the electricity market should not change the behaviour of electricity market actors such as producers and consumers

- Changes in the electricity market should have little effect on other markets.

Reliability cost comprises fixed and variable producer and TSO costs (GARPUR consortium 2016). Interruption costs are calculated based on the amount

\footnotetext{
${ }^{4}$ These two assumptions are never fully met. If, for example, electricity becomes more expensive and consumers' price elasticity is less than one, consumers will buy less electricity and will have less budget left to buy other goods.
} 
of load curtailed at a particular load point and value of lost load (VoLL) of interrupted customers, which amongst others depends on the time, duration and type of the interruption and the type of customer (GARPUR consortium 2016).

Complementary, technical indicators can be analysed, however, they cannot substitute total system cost in an overall performance evaluation, as they do not consider the reliability decision related trajectory. These technical indicators are useful to determine how close the system is operated to its limits if a particular reliability criterion is applied. Possible technical performance indicators might be the probability or magnitude of violating operational security limits or load curtailment. Operational security limits can be defined in terms of voltages, branch flows, etc.

Performance indicators can be expressed in terms of risk, which is defined as probability times severity, maximal, minimal or average values or probability density functions.

\section{PERFORMANCE EVALUATION TECHNIQUES FOR SHORT TERM RELIABILITY MANAGEMENT}

A hybrid performance evaluation technique following the analysis framework is presented. The technique combines aspects of simulation techniques and analytical contingency enumeration and is applied to short term reliability management. Short term reliability management aims at meeting a predefined reliability criterion (Heylen \& Van Hertem 2014). It consists of making decisions leading to preventive, corrective or no actions. Preventive actions are taken ahead of real time in order to achieve security and improve the ability to withstand possible effects of potential contingencies (GARPUR consortium 2014). As those actions are taken ahead of real time, decisions are based on expected system states that might differ from real time realizations due to contingencies and forecast errors of load and renewable power generation. Credible states considered in decision making ahead of real time are determined by the reliability criterion that is applied.

Corrective actions are taken in a final decision stage in real time using realizations of load, renewable generation and contingencies, while taking into account decisions taken in the preventive stage. Corrective actions allow to keep the system within limits and to satisfy the applied reliability criterion. The process of short term reliability management is graphically summarized in figure 2. The hybrid technique is compared with an analytical state enumeration approach in section 4.

\subsection{Hybrid performance evaluation technique}

The hybrid technique for performance evaluation used in this paper is a simulation technique using Latin Hypercube Sampling (LHS) for load and

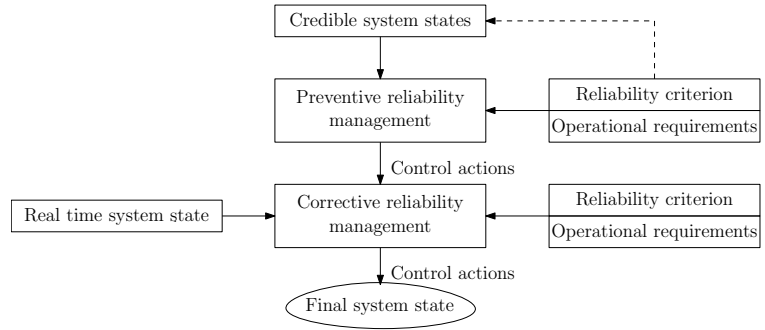

Figure 2: Overview of short term reliability management

RES uncertainty combined with analytical contingency enumeration. LHS allows to use less data points in a sample compared to random sampling and appears to be a good method to use for selecting values of input variables (McKay, Beckman, \& Conover 1979). The methodology is graphically illustrated for a 2-dimensional example in figure 3. LHS requires that variables are independent (Stein 1987). Principal component analysis (PCA) is used to determine independent components approximating the multidimensional, correlated probability structure. These independent and orthogonal components follow normal distributions of which the variances are given by eigenvalues $\lambda_{i}$ of the covariance matrix, as shown in figure 3(a). Sampling can be done along each principal component (PC) by dividing the probability axis of each dimension in multiple, equal intervals, as illustrated by figures 3(b), 3(c) and 3(d). A data point needs to be determined in each interval, within which a uniform distribution is assumed. This process is repeated for all considered independent dimensions leading to $\mathrm{N}$ multi-dimensional data points in terms of probabilities, which are transformed to principal component values using the cumulative probability density function. The resulting states are expressed in the coordinate system determined by the principal components, as shown by the blue crosses in figure 3(e). A matrix transformation using the considered principal components transforms the states to the original state space. This results in the states indicated by the black diamonds in figure 3(f). All states have equal probability of occurring, as sampling is based on the probability distributions.

Next to uncertainty regarding load and RES power generation, contingencies need to be considered. Contingencies are taken into account in an analytical way by evaluating all contingency cases up to a cumulative probability of $99.99 \%$ for all states determined by load and RES power generation in the sample.

Based on the results of evaluated states of load and RES power realizations, the hybrid technique allows to obtain the probability distribution of the weighted sum over the most probable contingencies up to a cumulative probability of $99.99 \%$ of total system cost. These sample results can be analysed statistically, e.g. using significance tests, confidence intervals, etc. 


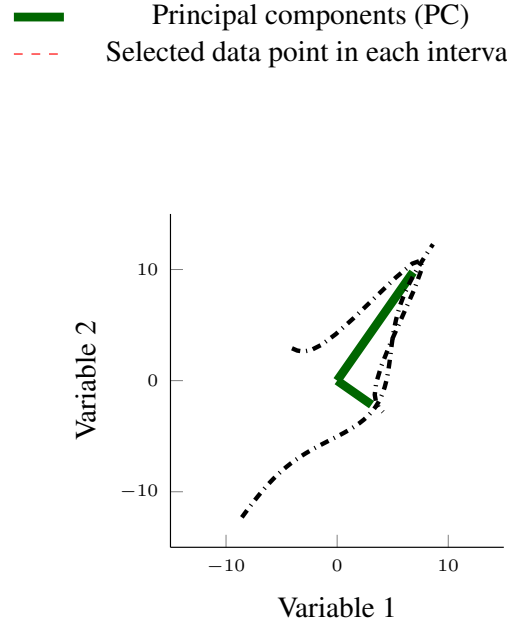

(a) PCA analysis of uncertainty cloud defined by correlation matrix and standard deviations of the variables resulting in 2 independent normal distributions $\times$-.. Shape normal distribution along PC
$\times$
lhs in PC coordinates
Interval boundaries

lhs after transformation
Probability

axis PC 2

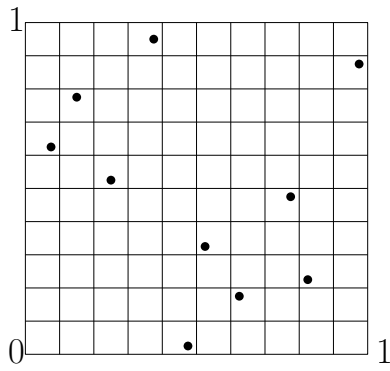

Probability axis PC 1

(b) Illustration of the procedure of Latin hypercube sampling along the probability axis of two principal components

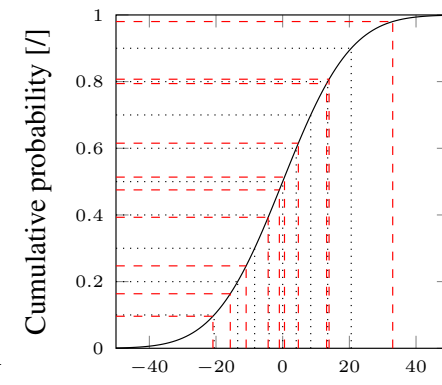

Principal component 1

(c) Sampling of first principal component along its cumulative probability axis and transformation to principal component coordinates using cumulative probability density function

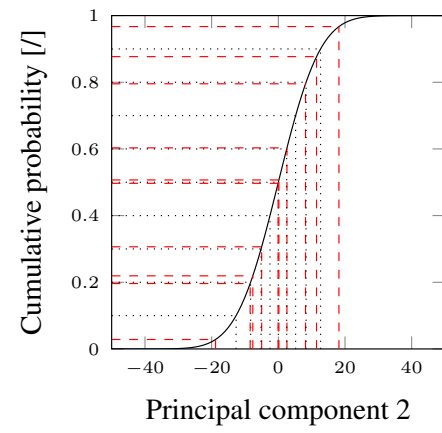

(d) Sampling of second principal component along its cumulative probability axis and transformation to principal component coordinates using cumulative probability density function

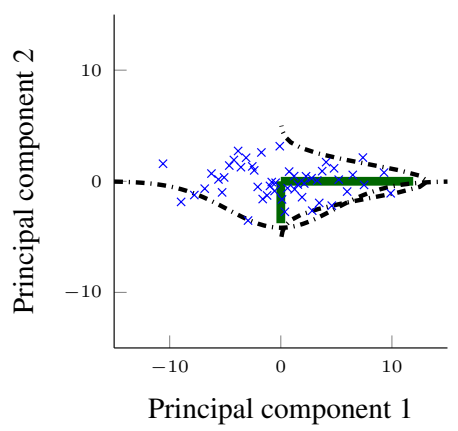

(e) Generated data points in principal component coordinates

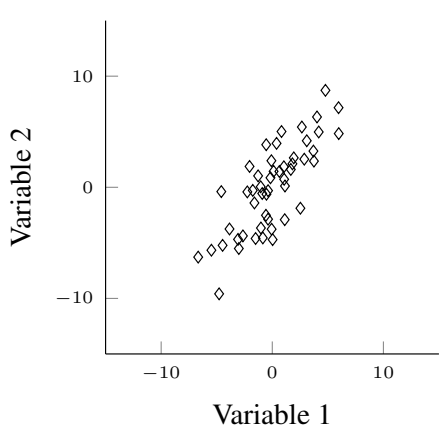

(f) Generated data points after transformation to original state space

Figure 3: Procedure of Latin hypercube sampling combined with principal component analysis illustrated for a two dimensional uncertainty cloud. Ihs refers to the Latin hypercube sample, while LHS refers to the Latin hypercube sampling procedure. The shapes of the probability density functions indicated in figures 3(a) and 3(e) can be compared, but the values corresponding to the height of the probability density function cannot be read on the graph. 
Three uncertainty levels are evaluated as summarized in table 2. Uncertainty is defined based on the generalized variance $(\mathrm{GV})$ of the covariance matrix relating uncertain load and wind power generation. Generalized variance is defined as the determinant of the covariance matrix and is proportional to the multi-dimensional volume of the ellipsoid approximating the uncertainty cloud (Templin 2005). The uncertainty level $U$ is defined as $\sqrt[n]{G V}$, which corresponds to the length of the side of a multi-dimensional cube of which the volume equals the generalized variance.

Table 2: Overview of the uncertainty levels in the different cases

\begin{tabular}{ccc}
\hline$U_{1}[\mathrm{MW}]$ & $U_{2}[\mathrm{MW}]$ & $U_{3}[\mathrm{MW}]$ \\
\hline 2.7 & 3.9 & 5.6 \\
\hline
\end{tabular}

Short term reliability management is simulated according to two reliability criteria:

- N-1 criterion: Load and wind power forecasts are used as expected real time load and wind power generation with a probability of 1 . Contingencies up to single branch outages are considered with equal probability. Load curtailment and operational limit violations need to be avoided in those contingency cases.

- Probabilistic reliability criterion: The objective is to minimize expected total system cost. Load uncertainty is taken into account in probabilistic reliability management ahead of real time using 7 load scenarios. These load scenarios are derived from a discretized normal distribution on top of the load forecast with a standard deviation of $4 \%$. The most probable contingencies up to a cumulative probability of $99 \%$ are considered. All system states are taken into account with their respective probability of occurrence.

Short term reliability management in the preventive and corrective stage is simulated using a stochastic DC security constrained optimal power flow (Heylen, Labeeuw, Deconinck, \& Van Hertem 2016, Zimmerman, Murillo-Sánchez, \& Thomas 2011). Generation redispatch is considered as preventive and corrective action. $20 \%$ of total system load is available for demand side response at a cost, while the remaining load can only be curtailed in emergency cases. The system is evaluated for a snapshot with a total load forecast of $165 \mathrm{MW}$ and a wind power generation forecast of 90 MW.

\subsection{Illustration}

Table 3 gives expected total system cost for various degrees of uncertainty according to the hybrid and analytical technique. The upper part of table 3 gives the results according to the $\mathrm{N}-1$ criterion, while the lower part focuses on the probabilistic reliability criterion. Expected total system costs according to the probabilistic and N-1 criterion are expressed relatively to
Table 3: Comparison of expected total system cost (ETC) for reliability management according to the $\mathrm{N}-1$ and probabilistic (Prob.) reliability criterion calculated with the hybrid (LHS) and analytical (ASE) technique for three uncertainty levels $U$ as defined in table 2. ETC is expressed relatively to the highest value obtained in the simulations, i.e. reliability management according to the N-1 criterion evaluated using analytical state enumeration.

\begin{tabular}{c|c|cc|cc|cc}
\hline & & \multicolumn{2}{|c|}{$U_{1}$} & \multicolumn{2}{|c|}{$U_{2}$} & \multicolumn{2}{|c}{$U_{3}$} \\
& & LHS & ASE & LHS & ASE & LHS & ASE \\
\hline \hline \multirow{2}{*}{$\mathrm{N}-1$} & ETC [\%] & 92.7 & 92.2 & 95.3 & 95.0 & 99.3 & 100 \\
& $\Delta_{\text {tech }}[\%]$ & -0.54 & -0.34 & \multicolumn{2}{|c}{0.66} \\
\hline \multirow{2}{*}{ Prob. } & ETC [\%] & 91.8 & 91.3 & 94.6 & 94.2 & 97.7 & 97.7 \\
& $\Delta_{\text {tech }}[\%]$ & -0.53 & -0.41 & \multicolumn{2}{|c}{0.02} \\
\hline
\end{tabular}

ASE: Analytical State Enumeration

the highest value obtained in the simulations, i.e. reliability management according to the $\mathrm{N}-1$ criterion evaluated using the analytical technique with uncertainty level $U_{3}$.

$\Delta_{\text {tech }}$ gives relative differences in expected total system cost (ETC) resulting from the hybrid (LHS) and the analytical state enumeration (ASE) technique at each uncertainty level and is defined as:

$\Delta_{\text {tech }}=100 \cdot \frac{\left(E T C_{A S E}-E T C_{L H S}\right)}{E T C_{L H S}}$

Differences in expected total system cost between both techniques are smaller than $1 \%$ for the considered uncertainty levels. The probabilistic reliability criterion outperforms the $\mathrm{N}-1$ criterion at the three uncertainty levels for both techniques.

\subsection{Comparison of performance evaluation techniques}

Bias of the evaluation techniques against particular reliability criteria needs to be avoided. Techniques might be biased if the credible system states prescribed by a particular reliability criterion and taken into account in the optimization of the preventive decision stage would match the evaluated system states. Evaluated contingency cases are selected in the same way in both techniques, namely the most probable contingencies up to a cumulative probability of $99.99 \%$. In this manner, additional contingencies are considered compared to the $\mathrm{N}-1$ and probabilistic criteria. More and different load and RES power realizations are also evaluated. The probabilistic criterion only assumes a normally distributed forecast error of total system load, while the N-1 criterion only takes into account the forecast value. In both performance evaluation techniques, variability of load and wind power generation at individual nodes is considered in the selection of system states to evaluate, as well as the correlation between those variables. The hybrid technique uses LHS of the uncertainty cloud, which results in random data points that are not related to the credible system states specified by the reliability criteria. The analytical technique divides the uncertainty 
cloud in intervals by applying PCA. The resulting intervals, of which the centre point is evaluated, are also not related to the credible system states specified by the reliability criteria. Sensitivity of the results to the selected evaluation states should be investigated leading to an efficient state selection technique.

Computation time is different for both techniques due to the number of system states that needs to be assessed. In the hybrid technique, a fixed number of 500 states is considered in the analysis for which the convergence in terms of total system cost is verified for the considered test case. However, in larger systems, the number of states might increase due to larger variabilities that might arise. The number of system states to evaluate in the analytical approach on the other hand is directly linked to the volume and shape of the uncertainty cloud, as this will determine the possibility for dimension reduction and the number of intervals. Moreover, computation time will increase in larger systems due to the increasing number of credible system states to consider in the simulation of reliability management.

In the presented illustration, only snapshots are assessed and temporal correlation between time steps is not considered. If these temporal aspects need to be included, sequential samples should be generated that take each interval in chronological order (Allan \& Billinton 2000). Sequential samples can be based on historical load and RES power profiles and allow to take into account constraints between time steps in the simulation, which increases the computational complexity. In order to obtain an efficient performance evaluation, a balance between increasing complexity of the simulations and accuracy is sought for.

\section{CONCLUSION AND FUTURE WORK}

Evaluating performance of power system reliability criteria is crucial to give incentives to power system stakeholders to apply cost effective and up-todate power system reliability management. Similarities exist between performance evaluation of power system reliability management and reliability assessment. However, reliability assessment mainly focuses on final system states, while performance evaluation should assess both the reliability decision related trajectory and consequences in final system states for an unbiased set of selected system states.

A key factor in the development or selection of an appropriate performance evaluation technique is the type of output information that is required, i.e. expected values or distributions. This is determined by the fundamental approach on which the evaluation technique is based, i.e. simulation, analytical or a combination of both (hybrid). A small case study for three uncertainty levels and short term reliability management according to two reliability criteria illustrates that a hybrid technique leads to similar results in terms of expected total system cost compared to an analytical state enumeration approach. Further work should focus on finding the optimal balance between computational complexity and accuracy.

\section{REFERENCES}

Allan, R. \& R. Billinton (2000). Probabilistic assessment of power systems. Proc. of the IEEE 88(2), 140-162.

Billinton, R., S. Kumar, N. Chowdhury, K. Chu, K. Debnath, L. Goel, E. Khan, P. Kos, G. Nourbakhsh, \& J. Oteng-Adjei (1989). A reliability test system for educational purposesbasic data. IEEE Trans. Power Syst. 4(3), 1238-1244.

CIGRE Working Group C4.601 (2010). Review of the current status of tools/techniques for risk based and probabilistic planning in power systems.

GARPUR consortium (2014). D2.1 functional analysis of reliability management. [Online]: http: //www.garpur-project.eu/deliverables.

GARPUR consortium (2016). D3.1 quantification method in the absence of market response and with market response taken into account. [Online]: http://www.garpur-project. eu/deliverables.

Heylen, E., G. Deconinck, \& D. Van Hertem (2015). Impact of value of lost load on performance of power system reliability criteria and their management. In Proc. IEEE PowerTech.

Heylen, E., W. Labeeuw, G. Deconinck, \& D. Van Hertem (2016). Framework for evaluating and comparing performance of power system reliability criteria (in press). IEEE Trans. Power Syst..

Heylen, E. \& D. Van Hertem (2014). Importance and difficulties of comparing reliability criteria and the assessment of reliability. In IEEE Young researchers symposium, Ghent. EESA.

Jolliffe, I. (2002). Principal component analysis. Wiley Online Library.

Khan, M. \& R. Billinton (1992). A hybrid model for quantifying different operating states of composite power systems. IEEE Trans. Power Syst. 7(1), 187-193.

Kjølle, G., O. Gjerde, \& M. Hofmann (2013). Vulnerability and security in a changing power system. Technical report, SINTEF Energy Research.

McKay, M. D., R. J. Beckman, \& W. J. Conover (1979). Comparison of three methods for selecting values of input variables in the analysis of output from a computer code. Technometrics 21(2), 239245.

Meliopoulos, S. (2005). Comprehensive power system reliability assessment. PSERC Publication 05-13, Georgia Institute of Technology.

Phadke, A. G. \& J. S. Thorp (1996). Expose hidden failures to prevent cascading outages. IEEE Trans. Comput. Appl. Power Syst. 11(3), 20-23.

Singh, C. \& J. Mitra (1997). Composite system reliability evaluation using state space pruning. IEEE Trans. Power Syst. 12(1), 471-479.

Stefopoulos, G. K., F. Yang, G. J. Cokkinides, \& A. S. Meliopoulos (2005). Advanced contingency selection methodology. In Proc. 2005 IEEE 37th Annu. North American Power Symp., pp. 67-73.

Stein, M. (1987). Large sample properties of simulations using latin hypercube sampling. Technometrics 29(2), 143-151.

Tamronglak, S. H. Horowitz, A. G. Phadke, \& J. S. Thorp (1995). Anatomy of power systems blackouts: Preventive relaying strategies. IEEE Trans. Power Del. 11, 708-715.

Templin, J. (2005). Generalized variance - multivariate normal distribution. [Online] Available: http://jonathantemplin.com/files/multivariate/ mv05psyc990/psyc990_04.pdf.

Warwick, K. (1997). Artificial intelligence techniques in power systems. IET.

Zimmerman, R. D., C. E. Murillo-Sánchez, \& R. J. Thomas (2011). Matpower: Steady-state operations, planning, and analysis tools for power systems research and education. IEEE Trans. Power Syst. 26(1), 12-19. 